\title{
Characterization of Ti-Ta-Sn Metallic Foams
}

Abraham Mejia ${ }^{1}$, Luis Bejar ${ }^{1}$, Luis Bejar-Vega ${ }^{1}$, Andres Bejar ${ }^{1}$, Claudio Aguilar ${ }^{2}$, Carolina Parra ${ }^{2}$ and Ismeli Alfonso ${ }^{3}$

${ }^{1}$ Universidad Michoacana de San Nicolás de Hidalgo, Morelia, Michoacan de Ocampo, Mexico, ${ }^{2}$ Universidad Técnica Federico Santa Maria, Valparaiso, Valparaiso, Chile, ${ }^{3}$ UNAM, Morelia, Michoacan de Ocampo, Mexico

The study of biomaterials today has developed a wide diversity of titanium-based metal alloys, in order to offer a better quality of life to people with different disabilities for bone tissue replacement [1]. One of the biomaterials most used in orthopedics is titanium, especially in the $\beta$ phase, due to its excellent mechanical properties and corrosion resistance [2-3]. It is also important to consider the elastic properties of the bone in a microstructural way which is between 10-30 GPa [4-5]. In the following work, a new TiTa-Sn metal alloy is studied as a biomaterial, the purpose of this work is to find a biocompatible material with the human body for medical implants, the alloy was manufactured by mixing powders, for 30 minutes, obtaining the products in green with a compression of $430 \mathrm{MPa}$. Heat treatment by a conventional method with a temperature curve of $25^{\circ} \mathrm{C}$ to $1200^{\circ} \mathrm{C}$ in an atmosphere controlled in argon for 6 hours. Ultrasound was performed to clean the impurity samples. X-ray diffraction (XRD). In fig.1 shows the diffractogram of the new phases generated by the metallic alloy of the Ti-Ta-Sn foam. The phases of Ti $\beta$ were identified with PDF 99-101-0425, Ta PDF99-101-0423, TiO2 PDF 99-100-4864, SnO2 PDF 99100-8913, and (Ti.5Ta.5) O2 PDF 99- 101-1709, the diffraction patterns obtained with the help of the Match software, the fig. 2 shows the morphology with microporosity at a magnification of 500X by electrodispersed electrons, the different shades of gray show a homogeneous mixture of elements used from the metal alloy of the new biomaterial. We can conclude the $\beta$ phase of titanium found in $38.51^{\circ}$, $55.59^{\circ}, 69.66^{\circ}, 82.52^{\circ}$ and $95.01^{\circ}$ angles $2 \Theta$ of the diffractogram of the Ti-Ta-Sn alloy, the characteristics that are presented up to the moment according to its crystalline structure which presents the beta phase and the formation of new phases which can be considered to obtain Good mechanical properties, which is a candidate for achieving an excellent biomaterial.

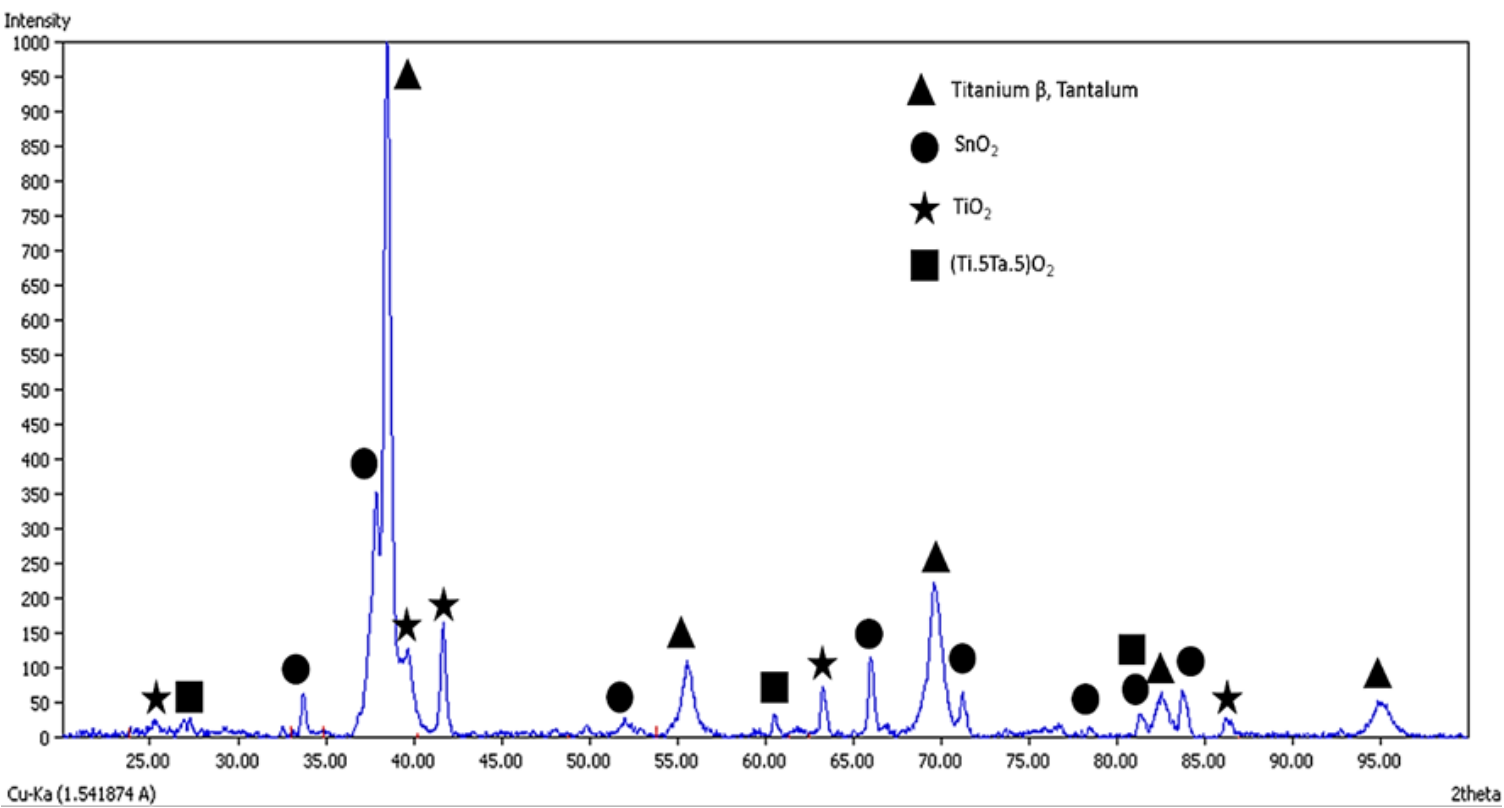


Figure 1. Diffraction patterns of Ti-Ta-Sn metal alloy foam.

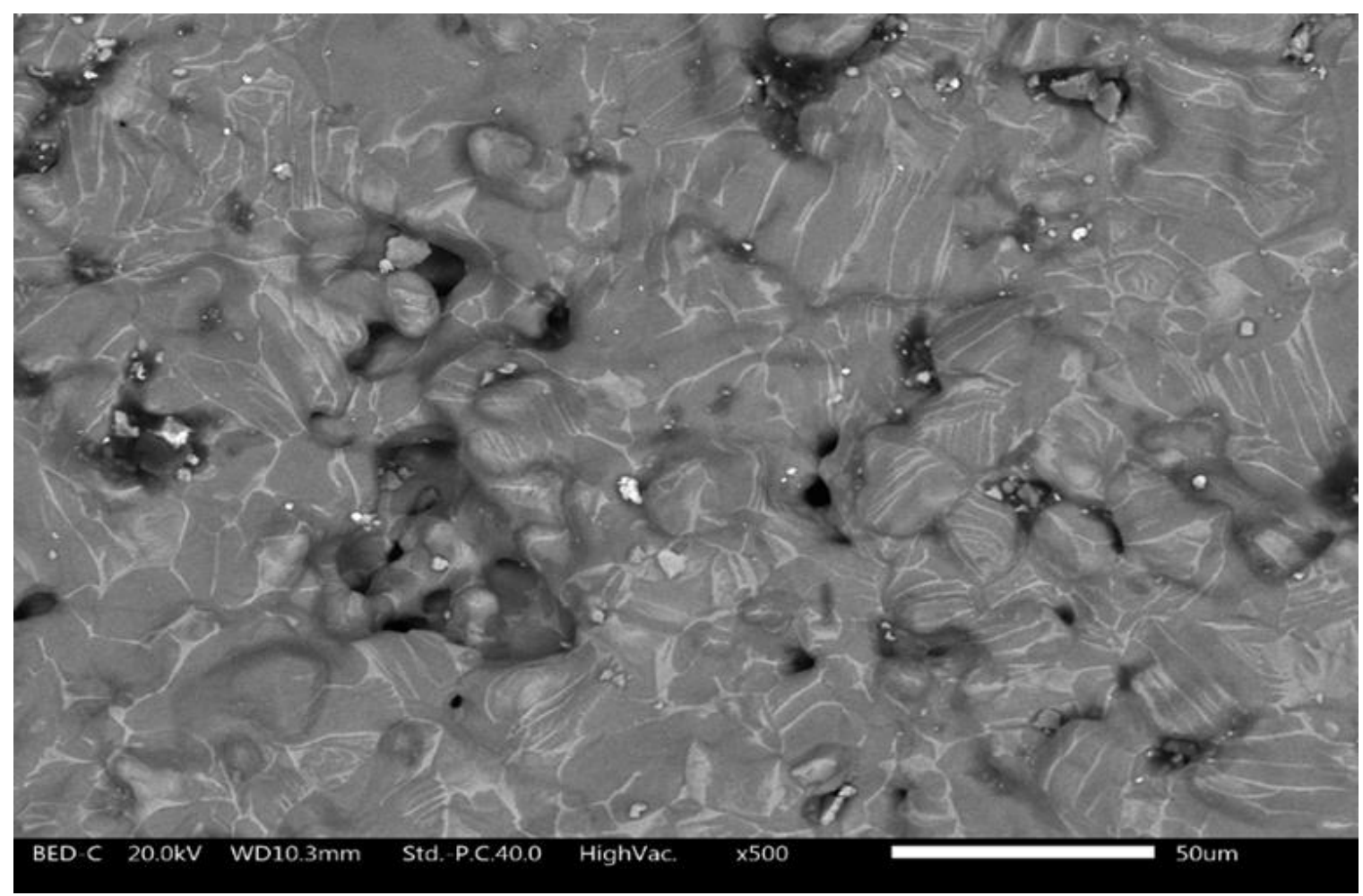

Figure 2. MEB image of the Ti-Ta-Sn metal alloy foam.

\section{References}

[1]. Torres, Y., Pavón, J. J., Nieto, I., \& Rodríguez, J. A. (2011). “Conventional Powder Metallurgy Process and Characterization of Porous Titanium for Biomedical Applications". Metallurgical and Materials Transactions B, 42(4), 891-900.

[2]. S.F. Jawed, C.D. Rabadia, Y.J. Liu, L.Q. Wang, Y.H. Li, X.H. Zhang, L.C. Zhang. "Mechanical characterization and deformation behavior of b-stabilized $\mathrm{Ti}$-Nb-Sn-Cr alloys". Journal of Alloys and Compounds 792 (2019) 684-693.

[3]. Mariana G. de Mello, Camilo F. Salvador, Alessandra Cremasco, Rubens Caram. "The effect of Sn addition on phase stability and phase evolution during aging heat treatment in Ti-Mo alloys employed as biomaterials". Materials Characterization 110 (2015) 5-13. 